\title{
The haptic perception of spatial orientations
}

\author{
Edouard Gentaz $\cdot$ Gabriel Baud-Bovy $\cdot$ Marion Luyat
}

Received: 24 July 2007 / Accepted: 7 April 2008 / Published online: 30 April 2008

(C) The Author(s) 2008

\begin{abstract}
This review examines the isotropy of the perception of spatial orientations in the haptic system. It shows the existence of an oblique effect (i.e., a better perception of vertical and horizontal orientations than oblique orientations) in a spatial plane intrinsic to the haptic system, determined by the gravitational cues and the cognitive resources and defined in a subjective frame of reference. Similar results are observed from infancy to adulthood. In 3D space, the haptic processing of orientations is also anisotropic and seems to use both egocentric and allocentric cues. Taken together, these results revealed that the haptic oblique effect occurs when the sensory motor traces associated with exploratory movement are represented more abstractly at a cognitive level.
\end{abstract}

Keywords Haptic - Space - Orientations - Oblique effect · Hand $\cdot$ Categorization $\cdot$ Reference frame $\cdot$ Development

E. Gentaz

National Center of Scientific Research

and University of Grenoble 2, Grenoble, France

G. Baud-Bovy

Faculty of Psychology, Vita-Salute San Raffaele University and IIT Network Research National Institute of Neuroscience, San Raffaele Foundation, Milan, Italy

\section{Luyat}

Laboratoire de Neurosciences Fonctionnelles et Pathologies (UMR CNRS 8160), Université de Lille, Service EFV,

Hôpital Roger Salengro, CHRU de Lille,

59037 Lille Cedex, France

E. Gentaz $(\square)$

Laboratoire Psychologie et NeuroCognition (UMR 5105),

Université Pierre Mendès France, Bâtiment des Sciences de

L'homme et des Mathématiques,

BP 47, 38040 Grenoble Cedex 9, France

e-mail: Edouard.Gentaz@upmf-grenoble.fr

\section{Introduction}

Haptic perception (or active touch) results from the stimulation of the mechanoreceptors in skin, muscles, tendons and joints generated by the manual exploration of an object in space (Gibson 1962; Revesz 1934, 1950). Haptic perception allows us, for example, to identify an object, or one of its features like its size, shape or weight, the position of its handle or the material of which it is made. A fundamental characteristic of the haptic system is that it depends on contact. The "tactile perceptual field" (i.e., the portion of the skin that is in contact with the external stimulus) has a limited size (the surface of both hands at maximum) and a limited reach (the length of the arm). It results from these characteristics of the tactile perceptual field that the perception of the spatial properties of the objects almost always involves some displacements of the arm and the hand to explore the stimulus. In fact, it is also known that the nature of these exploratory movements often depends on the specific property of the touched object (Lederman and Klatzky 1987, 1993). For example, one might follow the contour of an object with one finger to perceive its shape or squeeze it with the whole hand to perceive its compliance. Moreover, the haptic system must integrate information about the parts of the body touching the object with information about the position of the body parts in space. It results from these properties of the haptic system that the haptic perception of space is far removed from the proximal stimulation that occurs during the manual exploration, and depends on spatio-temporal integration of the kinesthetics and tactile inputs to build a representation of the stimulus.

Studies on the haptic perception of space have generally focused on the perception of the elementary spatial properties of objects such as their length, curvature or orientation in space (cf. Hatwell et al. 2003; Henriques and Soechting 
2005; Millar 2008). The hallmark of these studies is that the dimension of interest is systematically manipulated and that exploratory movements, if present, are very precisely monitored. In general, the aim of these studies is to measure the sensitivity of the corresponding perceptual channel. In addition, evidence such as a systematic bias or some variation in the sensitivity of parameters is used to understand the underlying processes. Altogether, these studies have shown that the haptic perception of simple spatial properties such as orientation and length are systematically distorted with respect to the physical reality. For example, it is well known that the haptic perception of the length between two points is influenced by numerous factors such as movement direction (radial versus tangential) or velocity (cf. Walsh et al. 1979; Imanaka and Abernethy 2002; Gentaz and Hatwell 2004). Others have found that straight lines are often perceived as curved and vice versa (Sanders and Kappers 2007; see also Henriques and Soechting 2003). In the same vein, Kappers $(1999,2002)$ and Kappers and Koenderink (1999) have observed that, two parallel (in physical space) bars in different spatial locations can be felt as being almost perpendicular. This remarkable effect remains even after various types of feedback. Finally, it is noteworthy that judgments of different spatial properties can be inconsistent among themselves. For example, Fasse et al. (2000) found that the biases in the perception of the acute angles of a right triangle were inconsistent with the observed biases in the judgments of the lengths of the adjacent and opposed edges. This study suggests that diverse spatial properties of an object might be processed independently from each other and do not necessarily refer to a unique underlying representation of space.

The present review is concerned by one very specific aspect of spatial perception: the haptic perception of spatial orientations. The perception of orientations is isotropic when the performances in orientation-adjustment tasks are similar regardless of the orientation values and anisotropic when performances differ according to these values. Historically, the orientation effect of a stimulus on the performance level was first studied in various detection, discrimination and reproduction tasks in the visual modality. A widespread observation, termed "oblique effect" by Appelle (1972), is that the performance level is in general better when the stimulus is aligned with the vertical or horizontal orientation. The original motivation for studying the oblique effect in the haptic modality was to test possible explanations of the visual oblique effect. The existence of an oblique effect in the haptic modality would suggest that orientations are handled by the same processes in both modalities and, thus, would reinforce the view that high-level, possibly amodal (i.e., independent of sensorial modalities), processes could be responsible for the oblique effect. In contrast, its absence would indicate the existence of distinct orientation-processing streams in the haptic and visual modalities (cf. Gentaz 2000; Gentaz and Hatwell 2004; Heller 2000; Millar 1994). In this respect, we will show that orientation processing is generally anisotropic in the haptic modality for adults, children and even in infants. However, unlike the visual oblique effect where such anisotropy is almost always present, we will show that this effect depends on the gravitational cues and memory constraints that are very specific to haptics.

The second objective of this review is to identify the frame of reference associated with the haptic oblique effect. This question comes from the observation that the concept of orientation is by definition relative to a set of fixed axes, which define a coordinate system or frame of reference. For example, in the polar coordinate system, an orientation is defined by the angle between some features of the stimulus such as its main axis and an axis of the coordinate system. While the choice of this set of axes can be arbitrary, it is common to choose the vertical and its perpendicular (the horizontal) because of the permanent influence of the vertical gravity force on Earth. In fact, the concept of verticality is so dominant that even in a drawing put on a table that is on a horizontal plane, the verticality is projected as the line belonging to the sagittal plane and perpendicular to the axis of the body. However, the status of verticality must be questioned. In the visual modality, various studies have examined whether the oblique effect was tied to this geocentric reference frame or to a retinocentric frame of reference. In this respect, we will show that the haptic oblique effect observed in the frontal plane is defined in a subjective reference frame that combines ego and geocentric cues. We will also raise the question of which pair of angles is used to code the orientation of a stimulus in space, when the stimuli do not necessarily belong to a plane, where a single angle would suffice to code the orientation. As a matter of fact, it is important to note that different pairs of angles can be used to code an orientation in space in the same way as different coordinate systems can be used to code the position of a point in space.

In conclusion, we will argue that the haptic oblique effect occurs at a relatively late stage of orientation processing, when sensory motor traces associated with exploratory movements are transformed into a more abstract representation of the orientation, because the experimental factors that modify the haptic oblique effect's strength either contribute to establishing such a representation (e.g. gravitational cues) or favor its use (e.g., memory constraints). Finally, we will discuss various hypotheses about how the processes involved in the recoding of the sensory-motor traces might yield an anisotropy in the perception or recollection of orientations. 


\section{Hypotheses on the origins of the oblique effects}

Numerous studies have shown that vertically or horizontally oriented visual stimuli are generally perceived with greater precision than those oriented obliquely. While the existence of the visual oblique effect is well established, the explanations of this effect in vision are still debated today (e.g., Baowang et al. 2003; Essock 1980; Gentaz and Ballaz 2000; Gentaz and Tschopp 2002; Meng and Qian 2005; Westheimer 2003). Although orientation processing mainly involves the lower visual areas such as the Lateral Geniculate Nucleus (LGN) and the visual primary cortex (V1), it is completed at higher levels in the extra-striate and nonvisual areas (e.g., Goodale et al. 1991). The multitude of subcortical and cortical areas involved in orientation processing raises the question of where the processes responsible for the visual oblique effect specifically act in the anatomofunctional steps of visual processing. Two general hypotheses have been advanced to explain two types of oblique effect.

The first hypothesis has been in general advanced to explain the so-called "Class 1 oblique effects," a term coined by Essock (1980), which are observed in tasks measuring the basic functional properties of the visual system (e.g., acuity and contrast). The hypothesis is that the visual oblique effect is generated at the lowest levels of the visual system, such as LGN and/or V1 (for a discussion about the major role of $\mathrm{V} 1$, see $\mathrm{Li}$ et al. 2003). This hypothesis is supported by the very specific neurophysiological characteristics of orientation-selective neurons in these areas. In particular, it has been shown that neurons sensitive to the vertical and horizontal orientations are more numerous, sensitive and/or narrowly tuned than neurons sensitive to the oblique orientations (e.g., Furmanski and Engel 2000; Li et al. 2003; Saarinen and Levi 1995). According to this hypothesis, the processes generating the visual oblique effect would therefore be specific to this modality.

In contrast, "Class 2 oblique effects" are observed in more cognitive tasks involving identifying, memorizing and categorizing orientations. Moreover, Class 2 oblique effects are influenced by factors, such as internal models (Morgan 1991), recognition (Heeley and Buchanan-Smith 1990) or attention (Shiu and Pashler 1992), that are not amenable to the characteristics of the early stages of visual processing. For example, the mere fact that an oblique effect is present in the reproduction of the orientation of two distant large dots is difficult to explain in terms of the properties of direction-sensitive neurons, since this stimulus in principle stimulates all orientations equally (Cecala and Garner 1986; Westheimer 2003). The second hypothesis is therefore that some oblique effects might occur not only at the low-level areas of the visual system such as LGN or V1 but also at higher level areas (from V2 to associative areas). This hypothesis suggests that the processes generating the visual oblique effect might not be specific to this sensory modality (Heeley et al. 1997). While some models of the Class 2 oblique effects make reference to neurophysiological mechanisms (e.g., McMahon and MacLeod 2003; Westheimer 2003), other explanations are formulated only in functional terms. For example, a widely shared idea is that orientations are represented in a reference frame having the vertical or horizontal orientations as principal axes at some later stage of visual processing. In this frame of reference, identification of these two orientations as principal axes can be carried out simply by identifying the stimulus with one of the two references axes, whereas the coding of oblique orientations would require the integration of information derived from both axes (Foster and Westland 1998; Gentaz and Hatwell 1996; Marendaz 1998; Regan and Price 1986). For example, the coding of oblique orientations might involve the computation of the ratio of the two values, which corresponds to the projections of the oblique on the references axes. According to this view, the oblique effect is caused by some additional processing needed to encode oblique orientations relatively to the reference axes. Alternatively, it has been suggested that all orientations are encoded with the same precision, but that participants have a remarkably accurate internal representation of the vertical and horizontal orientation, which can be used to produce the response when perceived orientation corresponds to these norms (Heeley et al. 1997). In other words, instead of assuming that some computation increases the error for oblique orientation, the higher level processes would bring additional information about the vertical and horizontal that decreases the variable error at the reference axes.

Another possibility, adopted by Huttenlocher et al., is that orientations are recorded in categorical terms (Huttenlocher et al. 1991, 1994; see also the work on Categorical Perception, Harnard 2003). According to the Category-Adjustment (CA) model, each orientation is categorized in one of the categories that partition the orientation space. This model was developed to explain the biases toward the diagonals observed in various positionand orientation-recall tasks (see also Spencer and Hund 2003; Haun et al. 2005). According to the CA model, the recall of an oblique orientation would be biased toward the closest diagonal because it would combine a low-level (presumably unbiased) representation of the orientation with the center of the category including the orientation, typically one of the four quadrants. In other words, the gist of the model is that the mental partitioning or labeling of the orientations into a limited number of categories is at the origin of systematic bias in the encoding and recall of an orientation. Finally, Spencer et al. have recently developed an alternative theoretical framework, 
the Dynamic Field Theory (DFT) of spatial cognition, to explain the presence of systematic biases in orientationrecall tasks (Spencer et al. 2006, 2007). The DFT is based on the dynamic properties of several topologically organized and interconnected layers of units and can provide insights about developmental processes such as the change in direction of the bias in orientation recall tasks between childhood and adulthood, and about real-time processes such as the increase of the recall bias with time. More specifically, the DFT includes a perceptual map that interacts with the environment, a working memory map and a long-term memory map. According to the DFT, repeated exposure to a reference axis results in the storage of this piece of information in the long-term memory, which can be at the origin of a shift of the stimulus position in the working memory even in absence of explicit contextual information in the environment. It is important to note that both the CA and DFT models were originally conceived to explain the results of experiments dealing with visual stimuli showing the presence of systematic biases toward the diagonals in various orientation or position recall tasks, a type of spatial anisotropy that, as we shall see, has not yet been studied in detail in the haptic modality. In the following section, we start to review experimental studies of the haptic oblique effect.

\section{Isotropic or anisotropic haptic perception of spatial orientations?}

Existence of an haptic oblique effect

Lechelt et al. (1976) were the first to show interest in the haptic oblique effect. The goal was to show the existence of an oblique effect in the haptic system analogous to the one observed in the visual system for the abovementioned reasons. To that end, they compared orientation perception in the visual and haptic systems by applying the same experimental paradigm to both systems. The visual and haptic stimuli were rods $(30 \mathrm{~cm} \times 0.6 \mathrm{~cm})$, which could take one of six orientations: $0^{\circ}, 45^{\circ}, 90^{\circ}, 135^{\circ}, 225^{\circ}, 315^{\circ}$. Adults were presented with two identical rods (a stimulus rod and a response rod) placed in the frontal plane, on either side and equidistant from their medial plane. In the visual condition, participants worked in a dark room with electroluminescent rods and gave verbal indications to adjust the response rod immediately after the stimulus rod had disappeared. In the haptic condition, participants were blindfolded and the response rod was adjusted with the contralateral hand immediately after the stimulus rod became unavailable.

Globally, the analysis of constant errors did not show any systematic deviation in either modality for all orienta- tions. The analysis of absolute errors revealed a classical effect of modality: errors were greater in the haptic condition (from $M=4.8^{\circ}$ to $M=10.5^{\circ}$ ) than those in the visual condition (from $M=1.5^{\circ}$ to $M=4.2^{\circ}$ ). The effect of spatial orientations was significant and revealed an oblique effect in both modalities. In vision, the errors were lower for vertical and horizontal $\left(M=1.5^{\circ}\right)$ than for the oblique orientations $\left(M=3.5^{\circ}\right)$. In haptics, the errors were lower for vertical and horizontal $\left(M=5^{\circ}\right)$ than for the oblique orientations $\left(M=9^{\circ}\right)$. Lechelt and Verenka (1980) replicated these results with a slightly modified procedure, where the rod exploration time was fixed at $5 \mathrm{~s}$ for both perceptual conditions, where the delay between exploration and reproduction was set at $10 \mathrm{~s}$, and where the orientations were no longer indicated by the experimenters before the test. The authors concluded that there was an oblique effect in the haptic system, analogous to that observed in the visual system.

Initial hypotheses on the origin of the haptic oblique effect: the role of prior knowledge and the mode of reproduction of the orientation

Appelle and Countryman (1986) questioned the abovementioned conclusions of Lechelt et al. (1976), and Lechelt and Verenka (1980). They argued that the haptic oblique effect might be induced by the prior visual perceptual experience and/or the conditions of exploration-reproduction movements. To show the possible effect of the first factor, the authors proposed two "prior experience" conditions: an "informed" condition, in which standard orientations were verbally and visually presented to participants for as long as they wished; and an "uninformed" condition, in which participants did not know which standard orientations were being tested. On the basis of Appelle and Gravetter's (1985) results, they predicted that prior verbal or visual knowledge of standard orientations would lead participants to produce responses using internal orientation models as a reference (mainly the vertical and horizontal orientations), instead of the immediate haptic percept resulting from the exploration of the stimulus, and induce the haptic oblique effect. With respect to the second factor (conditions of exploration-reproduction movements), these authors also argued that the haptic oblique effect observed in Lechelt et al.'s experiments could be due to the use of the contralateral hand to reproduce the orientation. Indeed, when one hand explores the orientation of a standard rod and the other hand reproduces the same orientation on a test rod, the symmetrical disposition of the shoulder-hand systems imposes nonhomologous movement patterns to explore and reproduce oblique orientations (agonist and antagonist muscles are mobilized differently). In contrast, exploration and reproduction movement patterns are homologous for 
vertical and horizontal orientations. Then, the authors proposed two exploration-reproduction conditions: contralateral condition (one hand explores and the other reproduces) and an ipsilateral condition (the same hand explores and reproduces). In the latter case, the exploration-reproduction movement patterns are homologous for all orientations and the oblique effect should not be present.

However, Appelle and Countryman (1986) modified the experimental paradigm of Lechelt et al. (1976), and Lechelt and Verenka (1980). First, they used only four orientations $\left(0^{\circ}, 45^{\circ}, 90^{\circ}\right.$ and $\left.135^{\circ}\right)$. Earlier results justified this choice, as they showed that performances did not differ between $45^{\circ}$ and $225^{\circ}$ or between $135^{\circ}$ and $325^{\circ}$, for example. They introduced decoy orientations so that participants would not be aware that only four orientations were being tested. They presented the orientations in the horizontal plane (parallel to a table surface) instead of the frontal one. Finally, they fixed the delay between exploration and reproduction at $5 \mathrm{~s}$. Absolute error analysis revealed that the performance on the oblique orientations, and therefore the amplitude of the haptic oblique effect, varied according to prior experience and exploration-reproduction conditions. Vertical and horizontal orientations were stable. The extent of the haptic oblique effect was maximal in the informed contralateral condition (vertical-horizontal: $M=5^{\circ}$ and obliques: $M=9^{\circ}$ ) and diminished in the uninformed contralateral condition (vertical-horizontal: $M=5.5$ and obliques: $M=7.5^{\circ}$ ). It was even smaller in the informed ipsilateral condition (vertical-horizontal: $M=3.5^{\circ}$ and obliques: $M=5.5^{\circ}$ ). Finally, it was nil, and the oblique effect thus absent, in the uninformed ipsilateral condition (vertical-horizontal: $M=4^{\circ}$ and obliques: $M=4.5^{\circ}$ ). In their study, Appelle and Countryman (1986) concluded that prior visual perceptual experience and exploration-reproduction movements were responsible for the haptic oblique effect, contrary to the propositions of Lechelt et al. (1976), and Lechelt and Verenka (1980).

Role of gravitational cues in the haptic oblique effect

Gentaz and Hatwell's (1995) study provides a test of Appelle and Countryman's (1986) hypothesis (second factor) regarding the existence of a difference between the onehanded exploratory movement patterns of an oblique orientation and the movement patterns needed to reproduce the oblique with the contralateral hand. As in Appelle and Countryman's (1986) study, the participants worked in a nonvisual and uninformed condition with a rod oriented at $0^{\circ}, 45^{\circ}, 90^{\circ}$ or $135^{\circ}$ and they reproduced the orientation of the standard rod after a 5-s delay. The standard rod was presented in the horizontal (as in Appelle et al.'s experiment), frontal (parallel to the surface of a painting, as in Lechelt et al.'s experiments) or sagittal (the median plane, perpen- dicular to the horizontal and frontal planes) planes. The task was performed ipsilaterally (with the same hand) or contralaterally (with the other hand). Given that, in the sagittal plane, the oblique orientation exploratory movements of one hand become homologous with those needed to reproduce the oblique orientations contralaterally; the oblique effect should also be absent in this plane if Appelle and Countryman's (1986) hypothesis (difference between exploration and reproduction movements) is determinant.

Absolute error analysis revealed that results did not concord with these predictions, because similar oblique effects were obtained in the frontal and sagittal planes under both ipsilateral and contralateral conditions (vertical-horizontal: $M=5^{\circ}$ and $45-135^{\circ}$ obliques: $M=8^{\circ}$ ). However, results in the horizontal plane replicated those of Appelle and Countryman (1986), i.e., an oblique effect was present in the contralateral condition (vertical-horizontal: $M=8.9^{\circ}$ and $45-135^{\circ}$ obliques: $\left.M=12.9^{\circ}\right)$ and absent in the ipsilateral one (vertical-horizontal: $M=6.3^{\circ}$ and $45-135^{\circ}$; obliques: $M=7.8^{\circ}$ ). Therefore, the presence or absence of an oblique effect does not appear to be due to the difference of movements between the two hands, but seems to be strongly linked to the type of spatial planes. To understand the results in the horizontal plane and, in particular, the lack of an oblique effect in the ipsilateral condition only, Gentaz and Hatwell (1995) asked blindfolded adults to symmetrically reproduce a previously presented orientation (a $45^{\circ}$ right standard oblique was to be reproduced at $45^{\circ}$ left and, similarly, a $45^{\circ}$ left standard oblique was to be reproduced at $45^{\circ}$ right) in the horizontal plane, either ipsilaterally or contralaterally. Given that the movements necessary for the exploration and the symmetrical reproduction of an oblique orientation become homologous in the contralateral condition and nonhomologous in the ipsilateral condition, the oblique effect should be present in the ipsilateral condition and absent in the contralateral condition if the Appelle and Contryman's hypothesis was valid. Results did not concord with these predictions, since an oblique effect was present in both reproduction conditions in the horizontal plane (vertical-horizontal: $M=7^{\circ}$ and $45-135^{\circ}$ obliques: $M=9.3^{\circ}$ ).

Taken together, these results partially (see below) invalidate Appelle and Countryman's (1986) hypothesis about the role of exploration-reproduction movements. Gentaz and Hatwell (1995) account for these results by suggesting that the haptic oblique effect was linked to the gravitational cues produced by the shoulder-hand system. Indeed, the need to relatively move massive parts of the body such as the arm and forearm during the manual exploration of a stimulus brings antigravity forces into play. The role that gravity might play in the haptic system is clearly reduced in the visual system. The shoulder-hand system is much more directly submitted to gravitational constraints than the ocular system. When it explores a stimulus, it has to produce 
antigravity forces and these, in return, provide what are known as "gravitational cues." These gravitational cues provided by the proprioceptive system result from the deformation of cutaneous, muscular and articular tissues, and depend on the specific muscular forces needed to maintain or displace the shoulder-hand system against gravity (Gentaz and Hatwell 1996). As such, these cues can provide a considerable amount of information on arm posture or motion, relative to some fixed "geocentric frame of reference" (Paillard 1991) that is linked to gravity and exterior to the body.

This hypothesis was justified by an analysis of the task conditions proposed in the previous experiments. Indeed, the size of the stimulus rod $(25 \mathrm{~cm})$ and its position in relation to the participant $(40 \mathrm{~cm})$ required significant participation of the shoulder-hand system during exploration. To explain the "plane effect" in the ipsilateral reproduction conditions observed above (i.e., the presence of an oblique effect in the frontal and sagittal planes and its absence in the horizontal plane), Gentaz and Hatwell (1996) hypothesized that participants favored gravitational cues in orientation processing, because these cues specify the gravitational vertical, which is used as a reference axis. The characteristics of these gravitational cues would differ according to exploratory conditions. In a normal situation, their average amplitude is practically the same in all three planes, since the arm is always influenced by the same gravitational forces. On the other hand, the variability of these cues is not the same depending on the plane the experiment is carried out in. There is a high variability in the frontal and sagittal planes, as the arm deploys considerable antigravitational forces when moving from bottom to top, but falls from top to bottom with little effort. On the contrary, in the horizontal plane, gravitational cues hardly vary during exploration as the arm constantly operates in a direction perpendicular to gravity.

Gentaz and Hatwell (1996) tested the hypothesis that the gravitational cues produced by the shoulder-hand system during exploration were involved in the oblique effect and in the "plane effect" in the ipsilateral reproduction condition. The amplitude of these cues was modified by manipulating the level of gravitational constraint, and variability was modified by changing the plane the task was effectuated in. The oblique effect and the plane effect should diminish in conditions where gravitational cues are weak, and increase in those where these cues are greater. In one experiment, only the horizontal plane was tested. Participants explored the orientation of a rod with one hand and reproduced it with the same hand, either keeping their forearm-wrist-hand in the air ("unsupported forearm" condition), or resting their forearm-wrist-hand on the rod supporting surface ("supported forearm" condition). In the first condition, antigravitational forces were necessarily produced, whereas these forces were very much reduced in the second condition. Absolute error analysis revealed that the oblique effect was absent in the supported forearm condition (vertical-horizontal: $M=4.7^{\circ}$ and $45-135^{\circ}$ obliques: $M=4.2^{\circ}$ ) and was present in the unsupported forearm condition (vertical-horizontal: $M=3^{\circ}$ and $45-135^{\circ}$ obliques: $M=7^{\circ}$ ). In another experiment, blindfolded adults were tested in one of the three planes, either in a "natural" condition, or in a "lightened" condition in which gravitational cues had been reduced by a pulley system. In the latter case, the participant's forearm was attached to a display, which held it balanced in the air when the weight suspended on the pulley reached a certain value variable from one participant to another. The amplitude of the oblique effect was lower in the lightened condition (significant difference of $1.43^{\circ}$ ) than that in the natural condition (significant difference of $3.43^{\circ}$ ), and this is because that the accuracy of vertical and horizontal reproduction was deteriorated (thus increasing the difference with that of the oblique orientations, which remained stable). In addition, the exploratory plane had no effect. Taken together, these results revealed that gravitational cues are crucial when the haptic oblique effect is present in the three planes in blindfolded participants and in the ipsilateral reproduction condition.

\section{Role of previous visual experience and memory} in the haptic oblique effect

The previous results do not, however, exclude an influence of high-level factors, such as visual experience or cognitive resources or both. As a matter of fact, it is well known that the haptic perception of the sighted working in a nonvisual condition can be fed by visual representations (Hatwell 1978; Thinus-Blanc and Gaunet 1997). Gentaz and Hatwell (1998) examined whether the participant's visual experience modified the action of the gravitational cues observed in sighted people. In this study, blindfolded sighted participants as well as early and late totally blind people were asked to explore a rod and to reproduce its orientation ipsilaterally. The magnitude of gravitational cues was modified by manipulating the level of gravitational constraints (natural and reduced) and their variability was modified by changing the task plane: horizontal (weak variability) and frontal (strong variability). By comparing the performances of the early and late totally blind, this study aimed at evaluating the role played by visual experience and visualization in the haptic oblique effect. If such is the case, the oblique effect should be present in the late blind and absent in the early blind. The comparison between blindfolded sighted and totally blind people, who are better-trained in the use of the haptic system, also allowed the authors to find out whether gravitational cues also played a role in the apparition of the haptic oblique effect in the blind as is the case 
with blindfolded persons. In this case, the oblique effect should be absent when cues are reduced and present when they are normal. Absolute error analysis showed that in the horizontal plane, the oblique effect was absent in both the early and late blind, regardless of gravitational constraints (vertical-horizontal: $M=7.9^{\circ}$ and $45-135^{\circ}$; obliques: $M=10^{\circ}$ ). In the frontal plane, the oblique effect was present in both groups of blind people, regardless of gravitational constraints (vertical-horizontal: $M=5.8^{\circ}$ and $45-$ $135^{\circ}$; obliques: $M=9.9^{\circ}$ ). Furthermore, no difference was observed between the early and the late blind. In conclusion, these results confirmed that the variability of gravitational cues plays an important role in the presence or absence of the haptic oblique effect in the totally blind, although no visual experience effect was observed [first factor of Appelle and Countryman's (1986) hypothesis].

Gentaz and Hatwell (1999) examined further the role of attentional and cognitive resources on the haptic perception of orientations and the oblique effect by increasing memorization constraints. We know that the characteristics of the haptic system differ in fundamental ways from those of the visual system. The limited size of the tactile perceptual field requires that it be moved to gain knowledge about, for example, the shape of an object. In contrast, eye movements play only a secondary role in the visual perception of the position or shape of an object, since both pieces of information can be extracted from static images. This difference was recently re-emphasized by Henriques and Soechting (2005) who argued in a recent review that "the processing of haptic information differs fundamentally from visual processing in that the former requires the integration of information that evolves in time as well as space." It results therefore from this quality of proximal reception of the haptic system that haptic processing and haptic perception are consistently more sequential than visual ones. Moreover, haptic perception needs to rely more heavily on working memory to achieve the mental synthesis that is necessary to gain an unified representation of the object (Revesz 1950; cf. Hatwell et al. 2003).

In all previous research on the haptic perception of orientations, the delay between the stimulus exploration phase and the reproduction phase was 5-s long and was unoccupied. In Gentaz and Hatwell (1999), memorization conditions were thus altered - the length and the nature of the gap was changed in the two exploratory conditions showing that the availability of gravitational cues affected orientation coding. Participants explored a rod in the horizontal plane with weak or natural gravitational cues and reproduced the rod's orientation ipsilaterally. The orientations were reproduced according to one of four memorization conditions: after unoccupied 5-s gaps or 30-s gaps and 30-s gaps occupied by interfering verbal or motor activities such as reciting the alphabet forwards or backwards (verbal) or haptically exploring a raised sinuous trajectory (motor). Absolute error analysis showed that when the gap was unoccupied (regardless of how long it was), haptic orientation treatment and the oblique effect depended on the exploratory conditions: the oblique effect was absent when gravitational cues were weak (vertical-horizontal: $M=5.5^{\circ}$ and $45-135^{\circ}$ obliques: $M=5.5^{\circ}$ ) and present when they were natural (vertical-horizontal: $M=3.85^{\circ}$ and $45-135^{\circ}$ obliques: $M=6.8^{\circ}$ ), like in Gentaz and Hatwell (1995, 1996). On the other hand, when the 30-s gap was occupied by interfering verbal or haptic activities, the haptic oblique effect was present in both experiments and regardless of available gravitational cues (vertical-horizontal: $M=5.2^{\circ}$ and $45-135^{\circ}$; obliques: $M=8.5^{\circ}$ ). Taken together, these results showed that high-level factors can indeed determine the presence of the haptic oblique effect, since the reduction of available attentional or cognitive resources by an interfering task caused an oblique effect in a condition in which it was not usually observed.

In conclusion, the presence of a haptic oblique effect in blindfolded sighted participants, as well as in the totally early blind, with natural exploration and ipsilateral reproduction in the frontal plane (regardless of gravitational constraints) invalidates Appelle and Countryman's (1986) hypothesis about the role of visual representations and shows the existence under very precise conditions of an oblique effect intrinsic to the haptic system (Table 1).

\section{Haptic orientations defined in a subjective reference frame}

As previously indicated, the concept of orientation is by definition relative to one or more systems of coordinates with the cardinal vertical and horizontal orientations as norms of this system. However, on Earth, different verticals (gravitational, egocentric), which can constitute the norms for independent references, exist. Thus, the existence of an oblique effect raises the question of which kind of vertical is better perceived or in other words in which reference frame this effect is defined. A classical broad distinction is made between egocentric (referred to the participant's body) and allocentric (referred to environmental cues) spatial frames (Howard 1982; Rock 1990). The allocentric reference frame can be divided into a gravitational frame defined by the direction of the gravity pull (geocentric frame) and in pattern-centric reference frames defined by (visual or haptic or both) contextual cues. In natural conditions, the different reference frames are in general congruent. Consequently, mapping of orientations could result from either egocentric or allocentric reference frames or most likely from both. In darkness, tilting the body uncouples 
Table 1 Average absolute errors in the studies of the haptic oblique effect with blindfolded adults

\begin{tabular}{|c|c|c|c|c|c|c|c|}
\hline \multicolumn{4}{|l|}{ Experimental conditions } & \multicolumn{2}{|c|}{ Orientations } & \multirow{2}{*}{$\begin{array}{l}\text { Oblique effect } \\
\text { Difference }\end{array}$} & \multirow[t]{2}{*}{ References } \\
\hline Tasks & Plane & Delay (s) & Hand & $\mathrm{V} / \mathrm{H}$ & Oblique & & \\
\hline \multicolumn{8}{|l|}{ Reproduction task } \\
\hline $\begin{array}{l}\text { Free-time exploration and haptically informed } \\
\text { about standard orientations }\end{array}$ & $\mathrm{F}$ & 0 & contra & 5 & 9 & $4^{*}$ & Lechelt et al. (1976) \\
\hline \multicolumn{8}{|l|}{ Reproduction task } \\
\hline \multirow{2}{*}{$\begin{array}{l}\text { Limited-time exploration }(5 \mathrm{~s}) \text { and haptically } \\
\text { informed about standard orientations }\end{array}$} & $\mathrm{F}$ & 0 & contra & 4 & 8.1 & $4.1^{*}$ & \multirow[t]{2}{*}{ Lechelt and Verenka (1980) } \\
\hline & $\mathrm{F}$ & 10 & contra & 4 & 7.5 & $3.5^{*}$ & \\
\hline \multicolumn{8}{|l|}{ Production task } \\
\hline $\begin{array}{l}\text { Free-time production and verbally } \\
\text { informed about orientations }\end{array}$ & $\mathrm{F}$ & 0 & one & 3.2 & 8.3 & $5.1^{*}$ & \multirow[t]{2}{*}{ Appelle and Gravetter (1985) } \\
\hline $\begin{array}{l}\text { Free-time and haptically } \\
\text { informed about orientations }\end{array}$ & $\mathrm{F}$ & 0 & one & 3.5 & 8.1 & $4.6^{*}$ & \\
\hline \multicolumn{8}{|l|}{ Reproduction task } \\
\hline Informed & $\mathrm{H}$ & 5 & Contra & 5 & 9 & $4^{*}$ & \multirow[t]{4}{*}{ Appelle and Countryman (1986) } \\
\hline Uninformed & $\mathrm{H}$ & 5 & Contra & 5.3 & 7.5 & $2.2^{*}$ & \\
\hline Informed & $\mathrm{H}$ & 5 & ipsi & 3.5 & 5.5 & $2^{*}$ & \\
\hline Uninformed & $\mathrm{H}$ & 5 & ipsi & 4 & 4.5 & 0.5 & \\
\hline \multicolumn{8}{|l|}{ Reproduction task } \\
\hline \multirow{5}{*}{$\begin{array}{l}\text { Free exploration and uninformed } \\
\text { about orientations }\end{array}$} & $\mathrm{F}$ & 5 & contra & 5 & 8 & $3^{*}$ & \multirow[t]{8}{*}{ Gentaz and Hatwell (1995) } \\
\hline & $\mathrm{F}$ & 5 & ipsi & 5 & 8 & $3^{*}$ & \\
\hline & $\mathrm{S}$ & 5 & contra & 5 & 8 & $3^{*}$ & \\
\hline & S & 5 & ipsi & 5 & 8 & $3^{*}$ & \\
\hline & $\mathrm{H}$ & 5 & contra & 8.9 & 12.9 & $4^{*}$ & \\
\hline Supported exploration & $\mathrm{H}$ & 5 & ipsi & 6.3 & 7.8 & 1.5 & \\
\hline Symmetric orientation & $\mathrm{H}$ & 5 & contra & 7 & 9.3 & $2.3^{*}$ & \\
\hline Symmetric orientation & $\mathrm{H}$ & 5 & ipsi & 7 & 9.3 & $2.3^{*}$ & \\
\hline \multicolumn{8}{|l|}{ Reproduction task } \\
\hline Unsupported exploration & $\mathrm{H}$ & 5 & ipsi & 3 & 7 & $4^{*}$ & \multirow[t]{8}{*}{ Gentaz and Hatwell (1996) } \\
\hline Supported exploration & $\mathrm{H}$ & 5 & ipsi & 4.7 & 4.2 & -0.5 & \\
\hline Normal gravity cues & $\mathrm{F}$ & 5 & ipsi & 3.5 & 8.2 & $4.7^{*}$ & \\
\hline Reduced gravity cues & $\mathrm{F}$ & 5 & ipsi & 6.1 & 7.2 & $1.1^{*}$ & \\
\hline Normal & $\mathrm{S}$ & 5 & ipsi & 3.6 & 7.1 & $4.5^{*}$ & \\
\hline Reduced & $\mathrm{S}$ & 5 & ipsi & 5.7 & 7.3 & $1.6^{*}$ & \\
\hline Normal & $\mathrm{H}$ & 5 & ipsi & 5.4 & 7.3 & $1.9^{*}$ & \\
\hline Reduced & $\mathrm{H}$ & 5 & ipsi & 7.6 & 9 & $1.4^{*}$ & \\
\hline \multicolumn{8}{|l|}{ Reproduction task Early and late blind Pooled } \\
\hline Normal & $\mathrm{F}$ & 5 & ipsi & 5.8 & 9.9 & $4.1^{*}$ & \multirow[t]{4}{*}{ Gentaz and Hatwell (1998) } \\
\hline Reduced & $\mathrm{F}$ & 5 & ipsi & 5.8 & 9.9 & $4.1^{*}$ & \\
\hline Normal & $\mathrm{H}$ & 5 & ipsi & 7.9 & 10 & 2.1 & \\
\hline Reduced & $\mathrm{H}$ & 5 & ipsi & 7.9 & 10 & 2.1 & \\
\hline \multicolumn{8}{|l|}{ Reproduction task } \\
\hline Normal, unoccupied delay & $\mathrm{F}$ & 5 & ipsi & 3.85 & 6.8 & $2.95 *$ & \multirow[t]{8}{*}{ Gentaz and Hatwell (1999) } \\
\hline Reduced, unoccupied delay & $\mathrm{F}$ & 5 & ipsi & 5.5 & 5.5 & 0 & \\
\hline Normal, unoccupied delay & $\mathrm{F}$ & 30 & ipsi & 3.85 & 6.8 & $2.95 *$ & \\
\hline Reduced, unoccupied delay & $\mathrm{F}$ & 30 & ipsi & 5.5 & 5.5 & 0 & \\
\hline Normal, verbal task & $\mathrm{F}$ & 30 & ipsi & 5.2 & 8.5 & $3.3^{*}$ & \\
\hline Reduced, verbal task & $\mathrm{F}$ & 30 & ipsi & 5.2 & 8.5 & $3.3^{*}$ & \\
\hline Normal, motor task & $\mathrm{F}$ & 30 & ipsi & 5.2 & 8.5 & $3.3^{*}$ & \\
\hline Reduced, motor task & $\mathrm{F}$ & 30 & ipsi & 5.2 & 8.5 & $3.3^{*}$ & \\
\hline
\end{tabular}


Table 1 continued

\begin{tabular}{|c|c|c|c|c|c|c|c|}
\hline \multicolumn{4}{|l|}{ Experimental conditions } & \multicolumn{2}{|c|}{ Orientations } & \multirow{2}{*}{$\begin{array}{l}\text { Oblique effect } \\
\text { Difference }\end{array}$} & \multirow[t]{2}{*}{ References } \\
\hline Tasks & Plane & Delay (s) & Hand & $\mathrm{V} / \mathrm{H}$ & Oblique & & \\
\hline \multicolumn{8}{|l|}{ Reproduction task } \\
\hline Upright & $\mathrm{F}$ & 5 & ipsi & 2.5 & 5.5 & $3^{*}$ & Luyat et al. (2001) \\
\hline Body tilted & $\mathrm{F}$ & 5 & ipsi & 5 & 5.5 & 0.5 & \\
\hline \multicolumn{8}{|l|}{ Production task } \\
\hline No context, smooth & $\mathrm{F}$ & 5 & ipsi & 2.3 & 6.4 & $4.1^{*}$ & Luyat et al. $(2005 a, b)$ \\
\hline Congruent haptic cues & $\mathrm{F}$ & 5 & ipsi & 1.4 & 3.4 & $2^{*}$ & \\
\hline Incongruent haptic cues & $\mathrm{F}$ & 5 & ipsi & 1.8 & 4.8 & $3.05 *$ & \\
\hline
\end{tabular}

The table reports the average absolute error for the vertical and horizontal orientations ( $V / H$ column), for the $45^{\circ}$ and $135^{\circ}$ orientations $(O b l i q u e$ column), as well as the existence of an oblique effect, that is, of statistically significant difference between the two sets of orientation (marked by * in the Difference column) as function of experimental conditions. The reported studies mainly used a reproduction paradigm where the subject explored the orientation of a rod with one hand before to be reproduced with the same (ipsilateral condition) or other hand (contralateral condition, see Hand column). The Plane and Delay column specify the plane in which the orientations were presented and reproduced or produced as well as the delay between the presentation and reproduction. The first column specifies additional experimental factors that were manipulated in the corresponding studies (see text)

the gravitational and the egocentric reference frames and allows us to specify in which kind of reference frame orientations are defined.

\section{Subjective reference frame}

In vision, certain studies have evidenced rather an egocentric (retinocentric) coding (Banks and Stolartz 1975; Chen and Levi 1996; Corwin et al. 1977), suggesting that the visual oblique effect could principally be accounted for by the properties of the orientation-selective neurons present in the primary visual cortex (see Introduction). Indeed, neurons tuned to vertical and horizontal retinal orientations are more numerous and feature particular response characteristics in relation to those tuned to oblique orientations. On the other hand, other studies have revealed the existence of gravitational orientation coding, implying that the origin of the oblique effect is probably more central, involving extraretinal information such as vestibular and somesthetic cues integrated at a higher level in the visual processing hierarchy (Attneave and Olson 1967; Buchanan-Smith and Heeley 1993; Ferrante et al. 1995; Lipshits and McIntyre 1999). However, very little research has been done concerning this topic in other perceptual modalities. In haptics, Luyat et al. (2001) tested the effect of tilting the body or the head on the haptic oblique effect. Blindfolded participants explored a standard rod in the frontal plane and reproduced its orientation with the same hand after a 5-s delay. Three conditions were examined: upright, inclined to the right $\left(+45^{\circ}\right)$ and to the left $\left(+135^{\circ}\right)$. Five orientations were tested: vertical, horizontal, $45^{\circ}$ oblique, $135^{\circ}$ oblique and the subjective vertical (SV). The SV is the individual's perception of the direction of gravitational force (cf. Howard
1982; Luyat 1997). The most commonly used paradigm consists of adjusting a stimulus (a rod) to the physical gravitational vertical. The deviation from the gravitational direction constituted a measure of the subjective vertical. In upright posture, the SV is very close to the physical vertical in participants with no vestibular disease or parietal lesion. However, in darkness, lateral head or body tilt provokes systematic deviations of the haptic SV in the opposite direction to that of the head: the Müller effect. A first experimental phase estimated each participant's SV in each postural condition and this SV was then tested in the exploration-reproduction task. What is more, as an insurance against a possible fluctuation of the SV in the haptic perception of orientation, the SV was measured again after the exploration-reproduction task.

If the haptic oblique effect is defined in a gravitational reference frame, then the reproduction of the vertical and the horizontal should be more accurate than the reproduction of oblique orientations, regardless of postural conditions. On the other hand, if the haptic oblique effect is defined in an egocentric reference frame, then the reproduction of the two diagonals $\left(45^{\circ}\right.$ and $\left.135^{\circ}\right)$, which are parallel and perpendicular to the body tilted to $45^{\circ}$ or $135^{\circ}$, should be more accurate than the reproduction of the gravitational vertical and the horizontal, which become oblique in relation to the body. Finally, knowing the effect of tilting the body on the vertical, the SV could constitute a reference axis when the body is tilted. In this case, the reproduction of the SV should be more accurate than all the other orientations in the tilted body conditions.

The results observed in the production task in which the participants had to manually orient the bar in the direction that they thought was vertical (phase 1, i.e., before the 
reproduction task and phase 2 , i.e., after the reproduction task) showed systematic deviations of the haptic SV in the direction opposite to that of the tilted body (Müller effect). The comparison of phases 1 and 2 showed that SV perception was stable and faithful over time, thus justifying its study in the exploration-reproduction task. Absolute error analysis in the exploration-reproduction task showed that tilting the body affects the precision of reproduction, particularly of the gravitational vertical and the horizontal. Thus, an oblique effect was present in the upright condition (vertical-horizontal: $M=2.5^{\circ}$ and $45-135^{\circ}$; obliques: $M=5.5^{\circ}$ ), but absent in tilted conditions (vertical-horizontal: $M=5^{\circ}$ and $45-135^{\circ}$ obliques: $M=5.5^{\circ}$ ). It should be noted that the same results were observed in a complementary experiment in which only the head was tilted.

In conclusion, tilting affected vertical perception in the production task and also in orientation reproduction. This means that, in a tilted body condition, the gravitational vertical and horizontal orientations no longer appear to act as reference norms, as they were not better reproduced than the oblique orientations. Consequently, the hypothesis of a pure gravitational reference frame underlying the haptic oblique effect can be rejected. In the same way, the results did not confirm the hypothesis of a pure egocentric reference frame, as $45^{\circ}$ (or $135^{\circ}$ ) oblique orientations (which are vertical or horizontal in relation to the body) were not better reproduced than the gravitational vertical and the horizontal. On the other hand, results showed that the SV was better reproduced than the other four orientations, particularly in the tilted body conditions $\left(M=2.7^{\circ}\right)$. In these conditions, the SV could constitute a norm for a subjective vertical reference frame.

Finally, these results questioned the nature of the processes responsible for the oblique effect across sensory modalities (Hatwell 1994; Thinus-Blanc and Gaunet 1997). Several studies in the spatial domain show that visual representations largely feed tactile representations and vice versa. Thus, Luyat and Gentaz (2002) examined whether the visual oblique effect is also coded in a subjective gravitational reference frame, analogous to that used in haptics. The effect of inclining the whole body on the visual oblique effect was studied with the same paradigm as that proposed in haptics. The classic oblique effect observed in the upright body condition disappeared in the inclined body conditions because of a reduction in the accuracy of the reproduction of the gravitational vertical and the horizontal. In inclined conditions, the subjective vertical appears to be the best perceived orientation. Thus, the visual oblique effect seems also to be defined within a subjective vertical reference frame. However, results in the production task showed that inclining the body produces systematic deviations to the visual subjective vertical (VSV) in the direction of the body incline axis, whereas deviation is in the direction opposite to body inclined in the haptic modality. In another study, Gentaz et al. (2001) examined the oblique effects in the visual, haptic and somato-vestibular systems by asking adults to reproduce (after a 10-s delay) an orientation presented in the frontal plane. In the visual modality, participants reproduced the orientation of a luminous rod presented in a dark room. In the haptic modality, blindfolded participants explored a rod with one hand and reproduced its orientation ipsilaterally. In the somato-vestibular modality, blindfolded participants reproduced the incline of their body. Results revealed similar oblique effects in the three tasks. However, no significant correlation between the magnitude of the visual, haptic and somato-vestibular oblique effects was observed. Taken together, these results suggest the existence of both processes specific to the haptic system (different subjective reference frames in haptics and in vision) and processes similar to the corresponding visual processes (access to a subjective reference frame) but not common across sensory modalities (absence of correlations between modalities). Let us now look at the effect of contextual cues on the haptic perception of orientation in adults by comparing it to the corresponding visual one.

\section{Haptic contextual reference frame}

In previous experiments, the stimulus consisted of a single rod without any contextual cues around it. In this case, it was not possible to determine whether the orientation perception in natural conditions could also be coded (at least partially) in a pattern-centric reference frame (defined by contextual cues). In vision, it is well known that spatial orientation is enhanced when vertical contextual cues are available, and as a consequence, it is strongly affected by roll or pitch of single tilted lines or by a more structured context such as a room (Groen et al. 2003; Luyat 1997). Recently, Luyat et al. (2005a) showed that the oblique effect decreased with visual contextual cues tilted $15^{\circ}$.

Another characteristic of the haptic system is that the shape and size of the tactile perceptual field can vary according to the mode of exploration adopted by the observer (e.g., with one or several fingers). This observation stands in sharp contrast with the visual system where the shape and size of visual field are essentially invariant. The ability of the haptic system to perceive contextual cues might vary drastically in accordance with the number of contacts that are established with the environment. For example, two-handed exploration might enlarge the haptic perceptual field sufficiently to facilitate the perception of contextual cues (on the role of reference cues, cf. Millar 1994; Millar and Al-Attar 2002).

The haptic perceptual field remains greatly reduced compared to the visual field even when the two hands are used with active exploratory movements. As a result, the 
perceptual cues, which are relevant in a task (or useful as a spatial exocentric reference frame), are less available in the haptic than in the visual modalities. Moreover, voluntary movements must be made to compensate for the smallness of the haptic perceptual field. As a result, haptic perception is highly sequential (cf. "Introduction"). However, this latter property permits contextual effects to be reduced in haptic perception compared to visual perception. Indeed, it is not the case in vision because of the presence of simultaneous peripheral (contextual cues) and central (the target) stimulation.

The effect of contextual cues on the perception of orientations was investigated first by Walker (1972) in a short report in which the participant had to position a rod to the vertical while passing the right hand through a tilted grid. This author reported an effect, but unfortunately both precise data and important methodological aspects as the amount of tilt used were not provided. More recently, Luyat et al. (2005b) studied this question by asking blindfolded participants to position a rod with their right dominant hand to three different spatial orientations in their fronto-parallel plane: to the vertical and along two oblique orientations, respectively, at $45^{\circ}$ to the left and to the right of the vertical one. This production task was carried out in four different backgrounds: (1) in the absence of contextual cues (smooth), (2) in presence of congruent contextual cues (stripes parallel to the orientation to be produced), (3) in presence of tilted stripes context to the left and (4) in presence of tilted stripes context to the right of the orientation to be produced. Using at the same time, the right and left hands with one hand exploring the context and the other (dominant hand) adjusting the stimulus-rod to the expected orientation allows a simultaneous stimulation by both the context and the stimulus.

The analysis of the precision of adjustments (variable errors) revealed that the oblique effect obtained in the control condition (no context) (vertical: $M=2.3^{\circ}$ and $45-135^{\circ}$ obliques: $M=6.4^{\circ}$ ) was similar to that obtained previously by Gentaz et al. (2002) with a similar method of direct estimation of orientations (production task). Indeed, most experiments on the haptic oblique effect have been carried out with a reproduction task, which requires memorizing an unknown but previously scanned orientation. This experiment confirmed that a haptic oblique effect in the frontoparallel plane can be evidenced with a production task, which involves a cognitive representation of orientations probably based on an internal model of gravity. More interesting is the fact that tactile contextual cues had an influence on the production of oblique and vertical orientations, particularly on the oblique effect. When the context was congruent with the standard orientations, the precision of adjustments was significantly improved (vertical: $M=1.8^{\circ}$ and $45-135^{\circ}$ obliques: $M=4.85^{\circ}$ ), an effect very similar to the effect exerted by visual vertical cues on spatial orientation. During noncongruent context scanning, the precision of orientations, particularly the obliques, also tended to be enhanced and, as a consequence, the oblique effect was significantly weakened. However, this improvement does not mean that the accuracy was enhanced, since analysis on constant error revealed that deviations erred in the direction of the stripes. Thus, the presence of a context, congruent or not, diminishes the variability of estimations and therefore enhances the precision of the production of orientations. Furthermore, the mismatch between the orientation to be produced and the context (noncongruent condition) revealed a tendency to adjust the rod in the direction of this context.

In conclusion, these results suggest that the haptic oblique effect is defined in a subjective reference frame, which may integrate not only vestibular and somesthetic inputs (head or whole body tilt) but also haptic contextual cues according to task conditions. Moreover, it should be emphasized that tasks such as pointing to remembered visual targets have also been found to involve processing information in multiple reference frames (see Carrozzo et al. 2002; McIntyre et al. 1997a, b). We propose that the subjective vertical and the subjective horizontal (probably) constitute the norms of this subjective vertical reference frame. At the neurophysiological level, the questions of how and where vestibular or contextual information dedicated to spatial cognition (specifically to the subjective perception of "what is up") is processed in the cortex is far from perfectly known. Research in humans with functional magnetic resonance imaging (fMRI) (Fasold et al. 2002) or electrical stimulation in patients with epilepsy (Kahane et al. 2003) have shown several vestibular cortex areas, in particular insulo-parietal (analogous to the Parieto-Insular Vestibular Cortex in monkeys) and temporal areas with a right hemispheric dominance. The integration of gravitational and contextual cues in the oblique effect suggests that these associative areas with multimodal neurons could be implicated. A last question raised in current haptic studies is the presence of the haptic oblique effect in $3 \mathrm{D}$ space in adults.

\section{Haptic orientation in 3D space}

In all previous experiments, we considered the haptic perception of orientations on a plane rather than in space. An orientation in space is defined by two parameters such as its azimuth and elevation. This observation raises the questions of whether the haptic oblique effect is still present in the absence of any spatial constraint when the participant needs to focus on at least two independent parameters to perform the task. More importantly, given the fact that there is a much larger set of alternative reference frames, 
which can be used to code an orientation in space than on a plane, the perception of orientations in 3D space raises the question of which reference frame is most adequate to describe the pattern of errors.

To examine this question, Baud-Bovy and Gentaz (2006) used a haptic display to present the stimuli and record the participant's hand movements to remove, if desired, the usual planar constraint imposed by the experimental apparatus used in most prior haptic studies. In a first experiment, the haptic reproduction of vertical, horizontal and diagonal orientations on a plane and in space was compared. Thus, participants were asked to explore the orientation of a "virtual rod" with to-and-fro movements on the frontal plane and then they were asked to respond in two different conditions. In the $2 \mathrm{D}$ reproduction condition, the finger movements were constrained to the frontal plane like in the exploration phase. In the 3D reproduction condition, the movements of the finger were unconstrained and could move freely in space. The first finding was that the vertical and horizontal orientations were better reproduced than the diagonal orientations in the 3D condition. Thus, removing the constraint during the reproduction phase did not prevent the occurrence of the haptic oblique effect. Decomposing the angular error in the 3D condition in an in-plane component and in an out-of-plane component revealed that the anisotropy in this condition concerned mostly the in-plane component. As a matter of fact, the error pattern in the 2D condition was very similar to the in-plane component of the $3 \mathrm{D}$ condition, while the analysis of out-plane errors showed that their distribution was much more uniform across orientations. This finding suggests first that an orientation in space is coded by two parameters (i.e., the angle inside the frontal plane and the angle between target orientation and the frontal plane) and second, that these two parameters are processed independently.

To examine how orientations are coded in space in the absence of any sort of reference to a plane, Baud-Bovy and Gentaz (2006) tested thirteen target orientations, which belonged to different planes (three principal axes-vertical, sagittal and lateral axes, the two $45^{\circ}$ and $135^{\circ}$ diagonals in the frontal, sagittal and horizontal plane, respectively, and four "3D diagonals"). As previously, blindfolded participants were asked to explore the orientation of a "virtual rod" with to-and-fro movements and to respond in the 3D condition, i.e., without any constraint on the finger movements during the reproduction phase. The haptic processing of orientations was clearly anisotropic. The vertical orientation was reproduced most precisely. Once again the accuracy and consistency with which participants reproduced this orientation was striking. For the other orientations, the error pattern depended in a complex manner on participants, stimulus and error components, which makes its interpretation complex.
On the one hand, the three principal axes were most accurately reproduced when all error components were pooled together. This observation has a straightforward consequence in a "plane-by-plane" analysis, as it implies that the horizontal and vertical axes in the frontal and sagittal planes, as well as the lateral and sagittal axes in the horizontal plane, are more accurately reproduced than the diagonal orientations. In other words, this observation is akin to a demonstration of the classic oblique effect in each one of these planes. Regarding the frontal plane, the results of this experiment were similar to those of the previous experiment. The analysis of the different error components showed a clear oblique effect in both experiments, with the vertical and horizontal orientations being most accurately reproduced, even in the absence of any planar structure in the design of the experiment and of any constraint during the reproduction phase. On the other hand, the presence of the classical oblique effect was much less obvious when we considered the various error components separately. The average angular error inside the horizontal and sagittal planes did not exhibit the expected pattern. Decomposing the in-plane errors in systematic, intersubject and intrasubject error components confirmed this negative finding. Further studies are needed to understand these complex patterns of results observed in 3D space. Let us now look at the ontogenetic development of the haptic oblique effect by comparing it to the corresponding visual one.

\section{Developmental aspect of the oblique effects}

The oblique effects in children and infancy

In vision, Leehey et al. (1975) tested the oblique effect in infants aged from 6 to 50 weeks using contrast sensitivity. The stimulus was a circular grating consisting of light and dark bars uniformly alternated. Bars were of five different widths. When spatial frequency was above threshold discrimination, the pattern appeared contrasted, whereas it looked like a homogeneous gray field when spatial frequency was below threshold. Two gratings of the same spatial frequency differing in orientations were simultaneously proposed. Each trial consisted of an oblique grating $\left(45^{\circ}\right.$ or $135^{\circ}$ ) and a vertical or a horizontal grating presented in a random fashion. As infants looked longer at the more contrasted of two patterns, a forced-choice judgment was used to determine which grating the young subject preferred. Given that the two stimuli have the same spatial frequency, an oblique effect would be present if, for a specific bar width, infants could discriminate the vertical or the horizontal lines but not the oblique lines. For this particular spatial frequency, infants should look preferentially at vertical or horizontal gratings rather than at oblique gratings. 
Results revealed an oblique effect. If the critical value of spatial frequency progressively decreased with age according to the development of visual acuity, an oblique effect was systematically observed at all ages. These findings were replicated by Gwiazda et al. (1978, 1984). According to Held and his colleagues, findings suggest that the visual oblique effect depends on endogenous maturation rather than exposure to a carpentered world, as assumed by other authors (Annis and Frost 1973; Ross 1992). Annis and Frost (1973) studied the oblique effect in Euro-Canadians raised in a carpentered environment characterized by the preponderance of cardinal contours, as well as in Cree Indians living in a traditional setting presenting more heterogeneous arrays of orientations. A classical matching task was used, subjects having to turn a test-rod until it matched the given standard-orientation. The four standard axes were tested: vertical, horizontal and the two diagonals. Prior knowledge of the tested orientations was given. The expected oblique effect was observed in Euro-Canadians, but it was absent in Cree Indians. In the same vein, Ross (1992) showed that its extent increases in an almost linear fashion between the ages of 7 and 12 years.

In haptics, the oblique effect has been studied in schoolage children and in infants. In children (from 6 years old to 10 years old), results showed that the oblique effect may be present under certain conditions (like in adults), and that its extent did not change (Gentaz and Hatwell 1995). In infancy, Gentaz and Streri (2002) initially examined whether the 5-month-old babies are capable of haptically discriminating (without visual control) between a vertical rod and a $45^{\circ}$ oblique rod positioned in the fronto-parallel plane. The authors offered each baby a 90 -s familiarization phase for one oriented rod. After this phase, a discrimination phase was proposed, in which the two orientations were alternately presented. By definition, haptic discrimination was said to occur when there was a significant difference between manual holding times in the discrimination phase for the familiar orientation (the one proposed in the familiarization phase) and for the novel orientation (for a discussion about the direction of preference, see Kerzerho et al. 2008). Results confirmed these predictions and thus showed that 5-month-old babies were capable of haptically discriminating between two orientations (vertical and $45^{\circ}$ oblique).

Then, Gentaz and Streri (2004) studied whether a haptic oblique effect is present from the age of 5 months. To answer this question, the authors used a method based on a critical angular value, as did Leehey et al.'s works in vision in babies. A haptic oblique effect is said to be present when the discrimination between two orientations does not only depend on their angular difference but also on the value of the tested orientation. In this case, the authors predicted that with the same angular difference $\left(10^{\circ}\right)$, discrimination should be observed between the vertical and a $10^{\circ}$ oblique orientation, whereas no discrimination should be observed between a $55^{\circ}$ oblique and a $45^{\circ}$ oblique. Results confirmed these predictions. These results showed that an oblique effect may be present when the shoulder-arm-hand system of the infants from the age of 5 months could actively move freely in the air to hold and to slightly explore the rod positioned in the frontal plane with the finger movements. This effect could be explained partially by the presence of the gravitational cues provided by the arm-hand system. These cues could reinforce gravitational (vertical) direction as an important axis, which could be used by infants as a reference axis to define spatial orientations, as it is the case in children and in adults (Gentaz and Hatwell 1996).

\section{Subjective-reference frame in infancy}

As in adults, the effect of body tilt on the oblique effect was investigated in the visual and haptic systems in infants. In vision, Jouen (1985) showed a classical oblique effect in the upright body position of 5-month-old infants, with longer looking at the vertical and horizontal gratings than at the $45^{\circ}$ and $135^{\circ}$ gratings. In contrast, in the tilted body position, infants looked longer at oblique stimuli aligned with their body orientation. These results showed that the visual oblique effect in infants is not defined in a gravitational reference frame but rather in a retinotopic reference frame. Consequently, this suggests that this effect would be directly related to the properties of orientation-selective neurons of the low-levels of the visual system where the retinotopical mapping of most orientation-selective neurons is preserved (cf. "Introduction").

In the haptic modality, Kerzerho et al. (2005) investigated the effect of whole body tilt on the haptic oblique effect by using the same method as Gentaz and Streri (2004). Two postural conditions were tested: upright body and body tilted $20^{\circ}$ to the left. Two orientations (vertical or $+20^{\circ}$-left oblique), defined gravitationally, were proposed in the familiarization phase and four (vertical, $10^{\circ}, 20^{\circ}$ or $30^{\circ}$-left oblique) in the test phase. The results showed that the body tilt had an effect on the infant's haptic discrimination of orientations. The understanding of this effect was complex, because it acted on both the spatial orientation discriminated and the direction preference of holding times. The pattern of results observed in the upright body position-the presence of a discrimination between vertical and $10^{\circ}$-left-oblique rods and a failure between $20^{\circ}$-left oblique and $30^{\circ}$-left oblique rods-confirmed Gentaz and Streri's (2004) previous observations and were in line with those found in vision by Held and his colleagues. However, the pattern of results observed in the tilted conditions showed the presence of discrimination between vertical and $10^{\circ}$ left-oblique rods and between $20^{\circ}$-left oblique and $30^{\circ}$-left 
oblique rods. In other words, the haptic oblique effect found in the upright posture disappeared when the body was tilted.

This effect of the body tilt on the patterns of results clearly showed that the hypothesis of a purely gravitational reference frame underlying the haptic oblique effect is not supported. Similarly, the results do not favor a purely egocentric reference frame, since a successful haptic discrimination was observed in the body tilted condition both (1) between the $20^{\circ}$ oblique rod that was vertical by reference to the body axis in the $20^{\circ}$ tilted position and the $30^{\circ}$ oblique rod and (2) between the gravitational vertical rod and $10^{\circ}$ oblique rod. In summary, the results suggest that spatial orientations are not defined only in a single reference frame. The results indicated that the haptic discrimination of the gravitational vertical (with the $10^{\circ}$ oblique) occurred in the two body positions. This result suggests that in tilted position, gravitational vertical seemed still to play the role of a reference axis at least partially. In contrast, the haptic discrimination of the $20^{\circ}$ oblique rod depended on the body position. It was absent in the upright position when the $20^{\circ}$ oblique rod was oblique by reference to the body axis, whereas it was present in the $20^{\circ}$ tilted body position when the $20^{\circ}$ oblique rod was vertical by reference to the body axis. This result suggests that, in the tilted position, the egocentric vertical also seemed to have the role of a reference axis, at least partially.

In conclusion, these results support the hypothesis of a reference frame, which integrates not only gravitational information but also egocentric information. As indicated before, this mixed-reference frame hypothesis underlying the orientation perception has already been proposed in the haptic (Kappers 2004) and visual (Lipshits et al. 2005; Lipshits and McIntyre 1999; Luyat et al. 2005a) systems in adults.

\section{General discussion and perspectives}

This review of experimental studies of the haptic oblique effect has showed that the perception of spatial orientations can be isotropic or anisotropic depending on various factors such as the presence of gravitational cues, the plane in which orientations are presented, the modality of response (ipsilateral or contralateral hand), etc. One of the most striking findings is the absence of an oblique effect in some conditions, which demonstrates that the haptic system can process all orientations isotropically. This observation stands in sharp contrast with vision where an oblique effect is observed much more systematically, probably because some form of directional anisotropy already emerges at the lowest levels of the visual system.

Our general interpretation of these studies is that the haptic oblique effect is a classic Class 2 oblique effect, which occurs relatively late in the processing of sensory information. More precisely, we believe that these studies globally indicate that the oblique effect occurs when the sensory motor traces associated with the exploratory movements are transformed into a more abstract representation at the cognitive level, where presumably the perceived orientation is related to a frame in reference that favors the vertical and horizontal orientations. While the high-level representation would introduce some degrees of anisotropy, it would also be less taxing from a memory point of view and be more robust to motor interference tasks. In this framework, gravity would facilitate the transformation from the low-level to the high-level representation by adding information about the direction of the movement relative to the vertical in the sensory-motor traces. In absence of these cues, the oblique effect would be smaller or absent, because the axes of the reference frames would be known with less certainty. In addition, studies conducted in the haptic and visual modalities where the geo-centered and ego-centered reference frames was dissociated by tilting the body have shown that the axes of this reference frame were influenced by vestibular and somesthetic inputs. Thus, these findings suggest that orientations might be represented in a frame of reference based on the subjective vertical and horizontal, that mixes ego- and geo-centric cues. While it is tempting to postulate that this subjective reference frame is shared between the two sensory modalities, a detailed analysis of the results revealed a lack of correlation between the sizes of the oblique effect obtained in each modality (Luyat et al. 2001; see detailed review in "Haptic orientations defined in a subjective reference frame'"). Additional studies are necessary to determine if this lack of correlation reflects only a difference of degree of anisotropy present in the visual and haptic processing of orientations or if it is due to a different positioning of the subjective axes in the modalities. In either case, the absence of a purely gravitational or egocentric frame of reference is a strong argument in favor of a high-level haptic oblique effect.

This review shows that gravity is a sufficient but not necessary condition for the occurrence of an oblique effect in the haptic modality. As a matter of fact, the haptic oblique effect can occur in absence of any gravitational cues. Moreover, the haptic oblique effect can also be strengthened by factors other than gravity. For example, some studies have shown the presence of an oblique effect with reduced gravitation cues when different hands were used in the exploration and reproduction phases (e.g., the contralateral condition in Appelle and Countryman 1986). To understand this result, it should be noted that the reproduction by the other hand of an oblique requires a complex transformation of the sensory-motor trace of the scanning movement given the symmetric arrangement of the two upper limbs. It is therefore plausible that the transformation from the lowlevel to the high-level representation is forced in this case, 
because the sensory-motor trace associated with the scanning arm is of little use for the reproduction phase and the haptic system must rely on the more abstract representation of the orientation (see Baud-Bovy and Viviani 1998 for a similar hypothesis in pointing context). Other studies have shown that an interference task during the delay between the presentation and the recall of the orientation (with the same hand) is responsible for an oblique effect in reduced gravity conditions or that it strengthens in normal conditions (Gentaz and Hatwell 1999). The idea is that higher attentional or cognitive demands decrease the amount of resources available to the haptic system. Such a situation would induce the transformation of sensory-motor trace or give more weight to a more abstract representation of the stimulus. Work in neighboring fields is consistent with this hypothesis. For example, Rossetti et al. (1996) showed that in proprioceptive pointing tasks, memorization leads participants to stop using sensory-motor representation to produce a movement and commits them to using a semantic spatial representation. In haptics, a similar shift in perception of parallelity was also observed by Zuidhoek et al. (2003). These authors tested the effect of a delay between the perception of a reference bar and the parallel setting of a test bar in the horizontal plane. They observed that a 10-s delay significantly improved performance. They suggest that a shift from the egocentric towards the allocentric reference frame during the delay period would lead to different spatial processing (for discussion, see also Faineteau et al. 2005; Gentaz and Gaunet 2006).

The nature of the more abstract representation and underlying processes is still an open question. One possibility is that the orientations would be mapped to one or more topologically organized layers of processing units, and that the perceived orientation would correspond to the most active units in one of these layers. The DFT of spatial cognition developed by Spencer and colleagues assumes such representational scheme (Spencer et al. 2006, 2007). According to this theoretical framework, the stimulus would propagate from perceptual maps to the workingmemory map, where the oblique effect would progressively occur under the influence of neighboring units and of the long-term memory map. While it is out of scope to enter into the details of this model here, it is important to note that the dynamic properties of these maps could explain the presence of a systematic bias toward or away from the reference axes as well as the increase of precision observed along the reference axes. Work on this model has so far focused on the visual modality and has not yet addressed issues specific to the haptic modality, such as the role of gravity or the coding of an orientation in space. In particular, the problem of extracting an initial representation of the orientation from the complex afferent and efferent signals that correspond to the sensory-motor trace of the scanning movement seems daunting, but this observation also holds true for all other explanations of the haptic oblique effect.

Another possibility is that the orientation would be represented in categorical terms (Huttenlocher et al. 1991; see also the work on Categorical Perception; Harnard 2003). As described in "Hypotheses on the origins of the oblique effects", the Category-Adjustment model assumes the existence of a double representation of the stimulus. According to the CA model, the oblique effect occurs at a late stage of processing, when both representations are combined, with possible different weights, to produce the response. From a theoretical point of view, such a double representation can be advantageous when the reliability of the low-level representation decreases, though it might introduce biases. In this theoretical framework, variations of the strength of the oblique effect could be understood in terms of a relative reliability of abstract representation of the sensory motor traces. The idea of a double representation could also be useful to explain the complex error patterns observed in the reproduction of 3D orientations showing that a participant responded using a heterogeneous set of cues that included the low-level sensory-motor trace as well as some more abstract-coding scheme based on the two angles between the orientation and the sagittal or frontal plane (Baud-Bovy and Gentaz 2006). However, a problem with the CA model is that it predicts an increase of the variability of response at the category boundaries (G. Baud-Bovy, 2008, A critical commentary of the category adjustment model and the oblique effect. Psychol Rev, unpublished). This observation is problematic insofar that it is usually assumed that the category boundaries correspond to the horizontal or vertical orientations. A possible remedy would be to assume the existence of narrow categories around the vertical and horizontal orientations in addition to the usual oblique categories centered on the diagonals.

To conclude, it is important to note that both the DFT and the CA model were originally conceived to explain the presence of systematic biases toward the closest diagonal in various location-recall or position-recall tasks in the visual modality, which has not yet been observed in the haptic modality. As a matter of fact, the analysis of the constant errors in studies of the haptic oblique effect did not reveal systematic directional errors. This observation holds true whether the reproduction method (Appelle and Countryman 1986; Gentaz and Hatwell 1995, 1996, 1998, 1999; Lechelt et al. 1976; Lechelt and Verenka 1980) or the production method (Appelle and Gravetter 1985; Gentaz et al. 2002; Luyat et al. 2001) was used to assess the perceived position of the orientation in the haptic modality. This negative result does not, however, invalidate these models, since the aforementioned studies of the haptic oblique effect have used a limited set of stimuli (the vertical, horizontal and the two main diagonals) that precludes the observation 
of a bias toward the closest diagonal, since it can be observed only with oblique orientations that differ from the diagonals. The question of the accuracy of the perception, as opposed to its precision, has been so far neglected in studies of the haptic oblique effect and needs to be further investigated in future studies.

Acknowledgments We thank Yvette Hatwell and Susan M. Campbell for their comments.

Open Access This article is distributed under the terms of the Creative Commons Attribution Noncommercial License which permits any noncommercial use, distribution, and reproduction in any medium, provided the original author(s) and source are credited.

\section{References}

Annis RC, Frost B (1973) Human visual ecology and orientation anisotropies in acuity. Science 182:729-731

Appelle S (1972) Perception and discrimination as a function of stimulus orientation: the "oblique effect" in man and animals. Psychol Bull 78:266-278

Appelle S, Countryman M (1986) Eliminating the haptic oblique effect: influence of scanning incongruity and prior knowledge of the standards. Perception 15:365-369

Appelle S, Gravetter F (1985) Effect of modality-specific experience on visual and haptic judgement of orientation. Perception 14:763-773

Attneave F, Olson RK (1967) Discriminability of stimuli varying in physical or retinal orientation. J Exp Psychol 74:149-157

Banks MS, Stolartz SS (1975) The effect of heald tilt on meridional differences in acuity: implications for orientation constancy. Percept Psychophys 17:17-22

Baowang L, Mattew RP, Ralph DF (2003) Oblique effect: a neural basis in the visual cortex. J Neurophysiol 90:204-217

Baud-Bovy G, Gentaz E (2006) The haptic perception of orientations in the frontal plane and in space. Exp Brain Res 172:283-300

Baud-Bovy G, Viviani P (1998) Pointing to kinesthetic targets in space. J Neurosci 18:1528-1545

Buchanan-Smith HM, Heeley DW (1993) Anisotropic axes in orientation perception are not retinotopically mapped. Perception 22:1389-1402

Carrozzo M, Stratta F, McIntyre J, Lacquaniti F (2002) Cognitive allocentric representations of visual space shape pointing errors. Exp Brain Res 147:426-436

Cecala AJ, Garner WR (1986) Internal frame of reference as a determinant of the oblique effect. J Exp Psychol Hum Percept Perform $12: 314-323$

Chen S, Levi DM (1996) Meridional anisotropy in the discrimination of parallel and perpendicular lines-effect of body tilt. Perception 25:633-649

Corwin TR, Moskowitz-Cook A, Green MA (1977) The oblique effect in a vernier acuity situation. Percept Psychophys 21:445-449

Essock EA (1980) The oblique effect of stimulus identification considered with respect to two classes of oblique effects. Perception 9:37-46

Essock EA, Krebs WK, Prather JR (1997) Superior sensitivity for tactile stimuli oriented proximally-distally on the finger: implications for mixed class 1 and class 2 anisotropies. J Exp Psychol Hum Percept Perform 23:515-527

Faineteau H, Gentaz E, Viviani P (2005) Factors affecting the size of the detour effect in the kinaesthetic perception of Euclidian distance. Exp Brain Res 163:503-514
Fasse ED, Hogan N, Kay B, Mussa-Ivaldi F (2000) Haptic interaction with virtual objects. Biol Cybernet 82:69-83

Fasold O, von Brevern M, Kuhberg M, Ploner CJ, Villringer A, Lempert $\mathrm{T}$ et al (2002) Human vestibular cortex as identified with caloric stimulation in functional magnetic resonance imaging. Neuroimage 17:1384-1393

Ferrante D, Gerbino W, Rock I (1995) Retinal vs. environmental orientation in the perception of the right angle. Acta Psychol $88: 25-32$

Foster D, Westland S (1998) Multiple groups of orientation-selective visual mechanism underlying rapid orientated-line. Proc $\mathrm{R}$ Soc Lond B Biol Sci B256:1605-1613

Furmanski C, Engel A (2000) An oblique effect in human primary visual cortex. Nat Neurosci 3:535-536

Gentaz E (2000) Existe-t-il un "effet de l'oblique" dans la perception tactile des orientations? Annee Psychol 100:111-140

Gentaz E, Badan M, Luyat M, Touil N (2002) The manual haptic perception of orientations and the oblique effect in patients with left visuo-spatial neglect. NeuroReport 13:327-331

Gentaz E, Ballaz C (2000) La perception visuelle des orientations et l'effet de l'oblique. Annee Psychol 100:715-744

Gentaz E, Gaunet A (2006) L'inférence haptique d'une localization spatiale chez les adultes et les enfants: etude de l'effet du trajet ct du délai dans une tâche de completion de triangle. Année Psychologique 106:167-190

Gentaz E, Hatwell Y (1995) The haptic "oblique effect" in children's and adults' perception of orientation. Perception 24:631-646

Gentaz E, Hatwell Y (1996) Role of gravitational cues in the haptic perception of orientation. Percept Psychophys 58:1278-1292

Gentaz E, Hatwell Y (1998) The haptic oblique effect in the perception of rod orientation by blind adults. Percept Psychophys 60:157167

Gentaz E, Hatwell Y (1999) Role of memorisation conditions in the haptic processing of orientations and the "oblique effect". Br J Psychol 90:377-388

Gentaz E, Hatwell Y (2004) Geometrical haptic illusion: role of exploratory movements in the Muller-Lyer, vertical-horizontal and Delboeuf illusions. Psychon Bull Rev 11:31-40

Gentaz E, Luyat M, Cian C, Hatwell Y, Barraud P-A, Raphel C (2001) The reproduction of vertical and oblique orientations in the visual, haptic, and somato-vestibular systems. Q J Exp Psychol 54:513526

Gentaz E, Streri A (2002) Infants' haptic discrimination of spatial orientations. Curr Psychol Lett 9:61-73

Gentaz E, Streri A (2004) An oblique effect in infants' haptic perception of spatial orientations. J Cogn Neurosci 16:1-7

Gentaz E, Tschopp C (2002) The oblique effect in the visual perception of orientations. In: Shovov S (ed) Advances in psychology research, vol vol 11. New York, Nova Sciences Publishers, pp $137-163$

Gibson JJ (1962) Observation on active touch. Psychol Rev 69:477491

Goodale MA, Milner AD, Jakobson LS, Carey DP (1991) A neurological dissociation between perceiving objects and grasping them. Nature 349:154-156

Groen EL, Jenkin HL, Howard I (2003) Perception of self-tilt in a true and illusory vertical plane. Perception 31:1477-1490

Gwiazda J, Brill S, Mohindra L, Held R (1978) Infant visual acuity and its meridional variation. Vision Res 18:1557-1564

Gwiazda J, Scheiman M, Held R (1984) Anisotropic resolution in children's vision. Vis Res 24:527-531

Harnard S (2003) Categorical perception. Encyclopedia of cognitive science. Nature Publishing Group/Macmillan

Hatwell Y (1978) Form perception and related issues in blind humans. In: Held R, Leibowitz HW, Teuber HL (eds) Handbook of sensory physiology, VII: perception. Springer Verlag, New York 
Hatwell Y (1994) Transferts intermodaux et intégration intermodale. In: Richelle M, Requin J, Robert M (eds) Traité de psychologie expérimentale. P.U.F, Paris, pp 543-584

Hatwell Y, Streri A, Gentaz E (2003) Touching for knowing. John Benjamins Publishing Compagny, Amsterdam

Haun D, Allen G, Wedell D (2005) Bias in spatial memory: a categorical endorsement. Acta Psychol 188:149-170

Heeley D, Buchanan-Smith H (1990) Recognition of stimulus orientation. Vis Res 30:1429-1437

Heeley D, Buchanan-Smith H, Cromwell J, Wright J (1997) The oblique effect in orientation acuity. Vis Res 32:235-242

Heller M (ed) (2000) Touch, representation and blindness. Oxford University Press, Oxford

Henriques D, Soechting JF (2003) Bias and sensitivity in the haptic perception of geometry. Exp Brain Res 150:95-108

Henriques D, Soechting JF (2005) Approaches to the study of haptic sensing. J Neurophysiol 93:3036-3043

Howard IP (1982) Human visual orientation. Wiley, New York

Huttenlocher J, Hedges L, Duncan S (1991) Categories and particulars: prototype effects in estimating spatial location. Psychol Rev 98:352-376

Huttenlocher J, Newcombe N, Hollister Sandberg E (1994) The coding of spatial location in young children. Cogn Psychol 27:115-147

Imanaka K, Abernethy B (2002) Distance-location interference in movement reproduction. In: Rossetti Y, Revonsua A (eds) Beyond dissociation: interaction between dissociated implicit and explicit processing. Advances in consciousness research. John Benjamins Publishing Company, Amsterdam

Jouen F (1985) The influence of body position on perception of orientations in infants. Behav Brain Res 15:241-245

Kahane P, Hoffmann D, Minotti L, Berthoz A (2003) Reappraisal of the human vestibular cortex by electrical stimulation study. Annu Neurol 54:615-624

Kappers A (2002) Haptic perception of parallelity in the midsagittal plane. Acta Psychol 109:25-40

Kappers AM (1999) Large systematic deviations in the haptic perception of parallelity. Perception 28:1001-1012

Kappers AM (2004) The contributions of egocentric and allocentric reference frames in haptic spatial tasks. Acta Pyschol 117:333340

Kappers AM, Koenderink JJ (1999) Haptic perception of spatial relations. Perception 28:781-795

Kerzerho S, Gentaz E, Streri A (2008) Factors influencing the manual discrimination of orientations in 5-month-old infants. Perception (in press)

Kerzerho S, Streri A, Gentaz E (2005) Reference frame and haptic discrimination of orientations in infants. NeuroReport 16:18331837

Lechelt EC, Eliuk J, Tanne G (1976) Perceptual orientational asymmetries: a comparison of visual and haptic space. Percept Psychophys 20:463-469

Lechelt EC, Verenka A (1980) Spatial anisotropy in intramodal and cross-modal judgements of stimulus orientations: the stability of the oblique effect. Perception 9:581-589

Lederman SJ, Klatzky RL (1987) Hand movements: a window into haptic object recognition. Cogn Psychol 19:342-368

Lederman SJ, Klatzky RL (1993) Extracting object properties through haptic exploration. Acta Psychol 84:29-40

Leehey SC, Moskowitz-Cook A, Brill S, Held R (1975) Orientational anisotropy in infant vision. Science 190:900-902

Li B, Peterson M, Freema R (2003) Oblique effect: a neural basis in the visual cortex. J Neurophysiol 90:204-217

Lipshits M, Bengoetxea A, Cheron G, McIntyre J (2005) Two reference frames for visual perception in two gravity conditions. Perception 34:545-555
Lipshits M, McIntyre J (1999) Gravity affects the preferred vertical and horizontal in visual perception of orientation. Neuroreport 10:1085-1089

Luyat M (1997) Verticale subjective versus verticale posturale: une note sur l'étude de la perception de la verticale. Annee Psychol 97:433-447

Luyat M, Gentaz E (2002) Body tilt effect on the reproduction of orientations: studies on the visual oblique effect and subjective orientations. J Exp Psychol Hum Percept Perform 28:1002-1011

Luyat M, Gentaz E, Corte T, Guerraz M (2001) Reference frames and haptic perception of orientation: body and head tilt effects on the oblique effect. Percept Psychophys 63:541-554

Luyat M, Mobarek S, Leconte C, Gentaz E (2005a) The plasticity of gravitational reference frame and the subjective vertical: peripheral visual information affects the oblique effect. Neurosci Lett 385:215-219

Luyat M, Moroni C, Gentaz E (2005b) The role of contextual cues in the haptic perception of orientation and the oblique effect. Psychon Bull Rev 12:760-766

Marendaz C (1998) Nature and dynamics of reference frames in visual search for orientation: implications for early visual processing. Psychol Sci 9:27-32

McIntyre J, Stratta F, Laquaniti F (1997a) Viewer-centered frame of reference for pointing to memorized targets in three-dimensional space. J Neurophysiol 78:1601-1618

McIntyre J, Stratta F, Laquaniti F (1997b) Short-term memory for reaching to visual targets : psychophysical evidence for body-centered reference frames. J Neurosci 18:8423-8435

McMahon M, MacLeod D (2003) The origin of the oblique effect examined with pattern adaptation and masking. J Vis 3:230-239

Meng X, Qian N (2005) The oblique effect depends on perceived, rather than physical, orientation and direction. Vis Res 45:3402-3413

Millar S (1994) Understanding and representing space. Theory and evidence from studies with blind and sighted children. Clarendon Press, Oxford

Millar S (2008) Space and sense. Psychology Press, Hove

Millar S, Al-Attar Z (2002) The Muller-Lyer illusion in touch and vision: implications for multisensory processes. Percept Psychophys 64(3):353-365

Morgan MJ (1991) Hyperacuity. In: Regan DM (ed) Spatial vision. The Macmillian Press, London, pp 87-113

Paillard J (1991) Brain and space. Oxford University Press, New York

Regan M, Price P (1986) Periodicity in orientation discrimination and the unconfounding of visual information. Vis Res 26:1299-1302

Revesz G (1934) System der optischen und haptishen Raumtaüschungen. Z Physiol 131:296-375

Revesz G (1950) Psychology and art of the blind. Longmans Green, London

Rock I (1990) The frame of reference. In: Rock I (ed) The legacy of Solomon Asch. Erlbaum, New Jersey, pp 243-268

Ross HE (1992) Orientation anisotropy: some caveats in interpreting group differences and developmental changes. Ophthalmol Physiol Opt 12:215-219

Rossetti Y, Gaunet F, Thinus-Blanc C (1996) Early visual experience affects memorization and spatial representations of proprioceptive targets. Neuroreport 7:1219-1223

Saarinen J, Levi D (1995) Orientation anisotropy in vernier acuity. Vis Res 35:2449-2461

Sanders D, Kappers AM (2007) Haptically straight lines. Perception 36:1682-1697

Shiu L-P, Pashler H (1992) Improvement in line orientation discrimination is retinally local but dependent on cognitive set. Percept Psychophys 52:582-588

Spencer JP, Hund AM (2003) Developmental continuity in the processes that underlie spatial recall. Cogn Psychol 47:432-480 
Spencer JP, Simmering VR, Schutte AR (2006) Toward a formal theory of flexible spatial behavior: geometric category biases generalize across pointing and verbal response types. J Exp Psychol Hum Percept Perform 32:473-490

Spencer JP, Simmering VR, Schutte AR, Schöner G (2007) What does theoretical neuroscience have to offer the study of behavioral development? In: Plumrt JM, Spencer JPS (eds) The emerging spatial mind. Oxford University Press, Oxford, pp 320-361

Thinus-Blanc C, Gaunet F (1997) Representation of space in the blind: vision as a spatial sense? Psychol Bull 121:20-42
Walker JT (1972) Tactual field dependance. Psychon Sci 26:311-313

Walsh WD, Russel DG, Imanaka K, James B (1979) Memory for constrained and preselected movement location and distance: effect of starting position and length. J Mot Behav 11:201-214

Westheimer G (2003) Meridional anisotropy in visual processing: implications for a neural site of the oblique effect. Vis Res 43:2281-2289

Zuidhoek S, Kappers AM, van der Lubbe RH, Postma A (2003) Delay improves performance on a haptic spatial matching task. Exp Brain Res 149:320-330 